\title{
Regulation and use of the extracellular matrix by Trypanosoma cruzi during early infection
}

\section{Pius N. Nde, Maria F. Lima, Candice A. Johnson, Siddharth Pratap and Fernando Villalta*}

Department of Microbiology and Immunology, School of Medicine, Meharry Medical College, Nashville, TN, USA

\section{Edited by:}

Wanderley De Souza, Universidade

Federal do Rio de Janeiro, Brazil

\section{Reviewed by:}

Renato A. Mortara, Escola Paulista de Medicina - Universidade Federal de São Paulo, Brazil

Tecia Maria Ulisses Carvalho, Universidade Federal do Rio de Janeiro, Brazil

\section{*Correspondence:}

Fernando Villalta, Department of Microbiology and Immunology, School of Medicine, Meharry Medical College, 1005 Dr. D.B. Todd Jr. Boulevard, Nashville, TN 37208, USA. e-mail: fvillalta@mmc.edu
Chagas disease, which was once thought to be confined to endemic regions of Latin America, has now gone global becoming a new worldwide challenge. For more than a century since its discovery, it has remained neglected with no effective drugs or vaccines. The mechanisms by which Trypanosoma cruzi regulates and uses the extracellular matrix (ECM) to invade cells and cause disease are just beginning to be understood. Here we critically review and discuss the regulation of the ECM interactome by T. cruzi, the use of the ECM by T. cruzi and analyze the molecular ECM/T. cruzi interphase during the early process of infection. It has been shown that invasive trypomastigote forms of $T$. cruzi use and modulate components of the ECM during the initial process of infection. Infective trypomastigotes up-regulate the expression of laminin $\gamma-1$ (LAMC1) and thrombospondin (THBS1) to facilitate the recruitment of trypomastigotes to enhance cellular infection. Silencing the expression of LAMC1 and THBS1 by stable RNAi dramatically reduces trypanosome infection. T. cruzi gp83, a ligand that mediates the attachment of trypanosomes to cells to initiate infection, up-regulates LAMC1 expression to enhance cellular infection. Infective trypomastigotes use Tc85 to interact with laminin, p45 mucin to interact with LAMC1 through galectin-3 (LGALS3), a human lectin, and calreticulin (TcCRT) to interact with TSB1 to enhance cellular infection. Silencing the expression of LGALS3 also reduces cellular infection. Despite the role of the ECM in T. cruzi infection, almost nothing is known about the ECM interactome networks operating in the process of $T$. cruzi infection and its ligands. Here, we present the first elucidation of the human ECM interactome network regulated by $T$. cruzi and its gp83 ligand that facilitates cellular infection. The elucidation of the human ECM interactome regulated by T. cruzi and the dissection of the molecular $\mathrm{ECM} / T$. cruzi interphase using systems biology approaches are not only critically important for the understanding of the molecular pathogenesis of T. cruzi infection but also for developing novel approaches of intervention in Chagas disease.

Keywords: laminin $\gamma-1$, thromospondin-1, calreticulin, gp83, ECM interactome, cellular infection, Trypanosoma cruzi, systems biology

\section{LAMC1 AND THBS1 ARE REQUIRED FOR T. cruZi INFECTION}

As part of our efforts to identify the molecular signatures induced by Trypanosoma cruzi in mammalian cells during the early infection process, we analyzed the kinetics of the extracellular matrix
(ECM) human transcriptome response. The result of this analysis showed that the only ECM proteins, LAMC1 and THBS1, were important in the early infection process. Accordingly, silencing the expression of LAMC1 and THBS1 by stable RNAi significantly
Abbreviations: 4A4, gp83 monoclonal antibody that neutralizes T. cruzi infection; ACHE, acetylcholinesterase (Yt blood group); APCS, amyloid P component, serum; APP, amyloid beta (A4) precursor protein (peptidase nexin-II, Alzheimer disease); ATF7IP, activating transcription factor 7 interacting protein; ATXN7L2, ataxin 7-like 2; B5, T. cruzi p45 mucin monoclonal antibody; BCAM, basal cell adhesion molecule (Lutheran blood group); BCL6, B-cell CLL/lymphoma 6; BIND, Biomolecular Interaction Network Database; BMP1, bone morphogenetic protein 1; bBTBD10, BTB (POZ) domain containing 10; C3, complement component 3; CALR or CRT, calreticulin; CCDC53, coiled-coil domain containing 53; CCSB-HI1, Center for cancer Systems Biology-Human Interactome; CD36, CD36 molecule (thrombospondin receptor); CD44, CD44 molecule (Indian blood group); CD47, CD47 molecule; $\mathrm{CFH}$, complement factor $\mathrm{H}$; cGMP, cyclic guanosine monophosphate; COL1A1, collagen, type I, alpha 1; COL2A1, collagen, type II, alpha 1; COL3A1, collagen, type III, alpha 1 (Ehlers-Danlos syndrome type IV, autosomal dominant); COL5A1, collagen, type V, alpha 1; COL6A1, collagen, type VI, alpha 1; COL7A1, collagen, type VII, alpha 1 (epidermolysis bullosa, dystrophic, dominant and recessive); CORO1A, coronin, actin binding protein, 1A; CSNK1A1, casein kinase 1, alpha 1 ;
CSNK2A1, casein kinase 2, alpha 1 polypeptide; CSNK2A2, casein kinase 2, alpha prime polypeptide; CTGF, connective tissue growth factor; CTSG, cathepsin G; CUBN, cubilin (intrinsic factor-cobalamin receptor); CYHR1, cysteine/histidinerich 1; DAG1, dystroglycan 1 (dystrophin-associated glycoprotein 1); DCN, decorin; DHFR, dihydrofolate reductase; DIP, Database of Interacting Proteins; E3T3C1, Cterminal domain of TSP-1, containing the last type II repeat domain, the three type III repeat domains and the carboxy-terminal domain; ECM, extracellular matrix; EGF, epidermal growth factor; ELA2, elastase 2, neutrophil; ELN, elastin (supravalvular aortic stenosis, Williams-Beuren syndrome); ERK 1/2, extracellular signal-regulated kinase; F2, coagulation factor II (thrombin); FBLN2, fibulin 2; FCGR2A, Fc fragment of IgG, low affinity IIa, receptor (CD32); FGA, fibrinogen alpha chain; FN1, fibronectin 1; GEMIN4, gem (nuclear organelle) associated protein 4; GFI1B, growth factor independent 1B transcription repressor; gp 83 or Tcgp83, T. cruzi trypomastigote ligand or trypomastigote surface $83 \mathrm{kDa}$ glycoprotein; HCASM, human coronary artery smooth muscle cell; HPRD, Human Protein Reference database; HRG, histidine-rich glycoprotein; HSPG2, heparan sulfate proteoglycan 2; INF- $\gamma$, Interferon gamma; IGFBP5, insulin-like growth factor 
reduced T. cruzi infection in cells (Nde et al., 2006; Simmons et al., 2006). The cells in which stable LAMC1 RNAi or THBS1 RNAi were performed showed significant reduction in the protein expression level of LAMC1 or THBS1 compared to cells stably transfected with vector alone or scrambled LAMC1 or THBS1 and the kinetics of T. cruzi infection were also dramatically reduced (Nde et al., 2006; Simmons et al., 2006). These studies showed that T. cruzi requires LAMC1 and THBS1 for early infection and indicated that host LAMC1 and THBS1 play critical roles in the early process of T. cruzi infection (Nde et al., 2006; Simmons et al., 2006). The important roles played by LAMC1 and THBS1 encouraged our group to dissect their molecular role in T. cruzi infection by looking at the ECM/T. cruzi interphase and elucidating the gene-networks and interactomes triggered by T. cruzi and its surface molecules involved in the early infection process (Cardenas

binding protein 5; IL-12, interleukin 12; IPI, International Protein Index; ITGA1, integrin, alpha 1; ITGA2, integrin, alpha 2 (CD49B, alpha 2 subunit of VLA-2 receptor); ITGA3, integrin, alpha 3 (antigen CD49C, alpha 3 subunit of VLA-3 receptor); ITGA4, integrin, alpha 4 (antigen CD49D, alpha 4 subunit of VLA-4 receptor); ITGA6, integrin, alpha 6 (alpha subunit of VLA-6 receptor); ITGAV, integrin, alpha $\mathrm{V}$ (vitronectin receptor, alpha polypeptide, antigen CD51); ITGB1, integrin, beta 1 (fibronectin receptor, beta polypeptide, antigen CD29 includes MDF2, MSK12); ITGB3, integrin, beta 3 (platelet glycoprotein IIIa, antigen CD61); ITGB4, integrin, beta 4; JAG1, jagged 1 (Alagille syndrome); KNG1, kininogen 1; KEGG, Kyoto Encyclopedia of Genes and Genomes; LAMA1, laminin, alpha 1; LAMA2, laminin, alpha 2 (merosin, congenital muscular dystrophy); LAMA3, laminin, alpha 3; LAMA5, laminin, alpha 5; LAMB1, laminin, beta 1; LAMB2, laminin beta 2 (laminin S); LAMB3, laminin, beta 3; LAMC1, laminin, gamma 1 (formerly LAMB2); LAMC2, laminin, gamma 2; LAMC3, laminin, gamma 3; LGALS3 lectin, galactoside-binding, soluble, 3; LGALS3BP, lectin, galactoside-binding, soluble, 3 binding protein; LIM2, lens intrinsic membrane protein 2, 19kDa; LRP1, low density lipoprotein-related protein 1 (alpha-2-macroglobulin receptor); LRP5, low density lipoprotein receptor-related protein 5; LTBP1, latent transforming growth factor beta binding protein 1; MATN2, matrilin 2; MEF, mouse embryo fibroblast; MEK, mitogen-activated protein kinase kinase; MEP1A, meprin A, alpha (PABA peptide hydrolase); MiMI, Michigan Molecular Interactions; MMP-2, matrix metallopeptidase 2 (gelatinase A, $72 \mathrm{kDa}$ gelatinase, $72 \mathrm{kDa}$ type IV collagenase) MMP-9, matrix metallopeptidase 9 (gelatinase B, $92 \mathrm{kDa}$ gelatinase, $92 \mathrm{kDa}$ type IV collagenase); MUC7, mucin 7, secreted; MYOC, myocilin, trabecular meshwork inducible glucocorticoid response; NID1, nidogen-1; NID2, nidogen-2 (osteonidogen); NO, nitric oxide; NT5E, $5^{\prime}$-nucleotidase, ecto (CD73); NTSP, N-terminal domain of TSP-1; ONECUT1, one cut homeobox 1; PDE1A, phosphodiesterase 1A, calmodulin-dependent; PDEA, phosphodiesterase 6A, cGMP-specific, rod, alpha; PDGFB, platelet-derived growth factor beta polypeptide (simian sarcoma viral (vsis) oncogene homolog); PLAT, plasminogen activator, tissue; PLC, phospholipase C; PLG, plasminogen; PPI, protein-protein interaction; PTPRF, protein tyrosine phosphatase, receptor type, F; RAF, v-raf-1 murine leukemia viral oncogene; RAS, rat sarcoma viral oncogene homolog; RNAi, RNA interference; RPSA, ribosomal protein SA; SAA1, serum amyloid A1; SAA2, serum amyloid A2; SCARB2, scavenger receptor class $\mathrm{B}$, member 2; SCD4, stearoyl-CoA desaturase 5; SDC1, syndecan 1; SDC3, syndecan 3; SIP1, survival of motor neuron protein interacting protein 1; SMAD2, SMAD family member 2; SNAPIN, SNAP-associated protein; SPARC, secreted protein, acidic, cysteine-rich (osteonectin); SPP1, secreted phosphoprotein 1 (osteopontin, bone sialoprotein I, early T-lymphocyte activation 1); SUFU, suppressor of fused homolog (Drosophila); SV2A, synaptic vesicle glycoprotein 2A; SV2B, synaptic vesicle glycoprotein 2B; SV2C, synaptic vesicle glycoprotein 2C; Tc45, T. cruzi $45 \mathrm{kDA}$ surface mucin; Tc85, T. cruzi $85 \mathrm{kDa}$ surface glycoprotein; TcCRT, T. cruzi calreticulin; TFPI, tissue factor pathway inhibitor (lipoproteinassociated coagulation inhibitor); TGF- $\alpha$, transforming growth factor alpha; TGF- $\beta$, transforming growth factor beta; TGFB1, transforming growth factor, beta 1; TSP1/THBS1, thrombospondin 1; TMPRSS6, transmembrane protease, serine 6; TNF $\alpha$, tumor necrosis factor alpha; TNFAIP6, tumor necrosis factor, alpha-induced protein 6; TNFRSF11B, tumor necrosis factor receptor superfamily, member 11b (osteoprotegerin); TNFRSF1A, tumor necrosis factor receptor superfamily, member 1A; TRAP; thrombospondin-related anonymous protein; TP53, tumor protein p53; TSC2, tuberous sclerosis 2; ZNF512B, zinc finger protein 512B; ZNF8, zinc finger protein 8 . et al., 2010; Nde et al., 2010). This will be critically reviewed in the next sections.

\section{T. cruZi SURFACE GP83 UP-REGULATES LAMC1 TO RECRUIT PARASITES AT THE ECM TO ENHANCE INFECTION}

Gp83 is a ligand expressed in all T. cruzi strains and employed by the parasite to attach and enter macrophages as well as nonphagocytic cells (Lima and Villalta, 1988; Villalta et al., 1998, 1999, 2001, 2008). Notably, it is expressed only in invasive trypomastigotes (Villalta et al., 1992) and is more expressed in highly infective trypomastigote clones (Lima and Villalta, 1989). Monovalent Fab fragments of the gp83-specific monoclonal antibody 4A4 inhibit gp83 binding to myoblasts, fibroblasts, and macrophages, block trypanosomes from attaching to and entering these cells, and neutralize T. cruzi infection in vivo (Villalta et al., 2001). Trypomastigotes release gp83 via parasite glycosylphosphatidylinositolphospholipase C (PLC) cleavage to activate the host MAPK pathway and PKC in order to promote parasite infection (Villalta et al., 1998, 1999; Nde et al., 2006).

Exposure of gp83 ligand to human cells increases the level of LAMC1 transcripts and its expression in mammalian cells, leading to an increase in cellular infection by T. cruzi (Nde et al., 2006). This increase in cellular infection was seen as over-attachment and entry of trypomastigotes into human cells over-expressing LAMC1, under the influence of gp83 ligand, resulting in high parasite multiplication at $72 \mathrm{~h}$. These observations together with the fact that knocking down the expression of LAMC1 by RNAi dramatically reduces $T$. cruzi attachment, entry and multiplication within cells strongly support the hypothesis that host LAMC1, which is regulated by the parasite gp 83, plays a crucial role in the early process of cellular infection.

The regulation of infection by the gp83 ligand represents a parasite escape mechanism in which invasive trypomastigotes release gp83 to efficiently gain entry into human coronary artery smooth muscle (HCASM) cells by manipulating LAMC1, which is the most abundant isoform of laminin in humans (Sasaki et al., 2004).

Trypanosoma cruzi must navigate through the basal lamina, which contains LAMC1, and surrounds individual muscle cells such as HCASM cells before infecting these cells. The fact that a T. cruzi trypomastigote ligand increases LAMC1 transcript levels in HCASM cells, correlates well with the finding that laminin is deposited in the hearts of patients infected with Chagas' disease (Milei et al., 1993). This suggests that the regulation of LAMC1 in heart cells by gp83 may be involved in heart pathology.

Since the T. cruzi gp83 ligand remodels the ECM by upregulating the expression of LAMC1, together with the report that $T$. cruzi presents laminin receptors on its surface (Giordano et al., 1999), indicates that the parasite exploits LAMC1 to navigate through the ECM, recruit trypanosomes and facilitate infection. Thus, the T. cruzi gp 83 ligand is a virulence factor that modifies LAMC1 expression in the ECM and contributes to the pathogenesis of T. cruzi infection in human heart cells.

\section{T. cruzi MODULATES THE LAMC1 SUB-NETWORK}

Recently, the first LAMC1 sub-network modulated by invasive trypomastigotes during the early process of infection was elucidated (Nde et al., 2010). To populate and build the interaction 
network, the Michigan Molecular Interactions (MiMI) cytoscape plugin (version 3.2) was used. MiMI retrieves molecular interactions from MiMI database and displays the interaction network with cytoscape. MiMI gathers and merges data from well-known protein interaction databases including BIND, DIP, HPRD, RefSeq, SwissProt, IPI, and CCSB-HI1. The plugin also integrates with other NCBI tools for literature information, document summarization, and pathway matching (Gao et al., 2009). The RT-PCR verified transcripts were used as the initial population nodes. MiMI was used to query for the initial nodes and their respective nearest neighbors to one degree. The networks were then merged for interconnections and the interactome was visualized in cytoscape (version 2.6.1) using the yFiles: organic layout format (Cline et al., 2007).

The first elucidated LAMC1 sub-network interactome mobilized in cells by $T$. cruzi involves the following genes CCDC53, NID1, NID2, SNAPIN, LAMA1, SV2A, CD44, LAMA5, ATF71P, ITGB4, LAMB1, LAMB2, SV2C, SV2B, ITGA6, and BCL6 (Nde et al., 2010). Our group is fully investigating the roles of the members of this sub-network in the infection process. Accordingly, the ITGA6 gene that encodes integrin alpha- 6 that combines with beta 4 as a laminin receptor, or with beta 1 in the integrin VLA- 6 participates in cell adhesion as well as cell-surface mediated signaling. The integrin, beta 4 (ITGB4) gene that encodes the integrin beta 4 subunit, a receptor for the laminins, tends to associate with alpha 6 subunit and appears to be involved in cell invasion. Entactin, also known as nidogen-1 (NID1) is a component of the basement membrane that connects the networks formed by collagens and laminins to each other and plays a role in cell interactions with the ECM. Furthermore, LAMA1 (Laminin, alpha 1) interacts with Fibulin-2 (FBLN2), an ECM protein that binds various extracellular ligands and calcium and appears to be involved in the infection process. Interestingly, BCL6 is a central hub of the interaction LAMC1 sub-network, which is also a common central hub to five of the seven sub-networks reported by one degree of connection to the initial seed nodes (Nde et al., 2010).

The elucidation of the LAMC1 sub-network interactome mobilized by T. cruzi is critically important to understand the molecular pathogenesis of T. cruzi infection derived from the ECM/T. cruzi gp83 ligand interplay and for the development of approaches for intervention. We expect that continuing to explore systems biology in the early process of $T$. cruzi infection will rapidly bring novel approaches for the treatment and management of Chagas disease.

\section{THBS1 INTERACTS WITH T. crUZI SURFACE CALRETICULIN TO ENHANCE CELLULAR INFECTION}

Thrombospondins have been described as "matricellular proteins" because they play a role in regulating cellular responses and ECM remodeling in the pericellular microenvironment but they are non-essential components of the mature matrix fibrils (Bornstein, 2002). The role of THBS1 in vitro and in vivo is complex and context specific, because it interacts with a wide array of cellular proteins. THBS1 is a large homotrimeric glycoprotein containing several domains that can bind to cell surface receptors and extracellular molecules (Chen et al., 2000) such as the $\mathrm{N}$-terminal heparin-binding domain (NTSP), procollagen region, type 1, 2, and 3 repeats and a C-terminal domain (Elzie and
Murphy-Ullrich, 2004). The molecule also contains highly conserved epidermal growth factor (EGF) repeats, type 3 repeats and a C-terminal domain, which includes the signature domain (Carlson et al., 2005) that can interact with integrins and CD47 (Lawler and Hynes, 1989; Gao and Frazier, 1994). The C-terminal domain of the thrombospondin family is highly conserved compared to the $\mathrm{N}$-terminal domain, which is different for each thrombospondin isoform.

Calreticulin (CRT) is a major intracellular well-conserved calcium-binding chaperone, which was identified in skeletal muscle (Michalak etal., 1992) and is present in the cells of all higher organisms except erythrocytes (Michalak et al., 1999; Johnson etal., 2001a,b). Numerous reports have implicated CRT in several cellular functions and the molecule has significant non-endoplasmic reticulum functions in normal physiology and human disease status (Gold et al., 2010).

Calreticulin has also been described in some parasite species such as Schistosoma mansoni, Onchocerca volvulus, Necator americanus, Leishmania donovani, and Plasmodium falciparum. However, the role that this protein might play in parasite interactions with the host immediate microenvironment remains unknown (Nakhasi et al., 1998; Ferreira et al., 2004, 2005).

Previous studies from our group showed that invasive T. cruzi trypomastigotes up-regulate the expression of THBS1 in HCASM cells (Simmons et al., 2006). Furthermore, knockdown of THBS1 rendered mammalian cells less susceptible to cellular infection by T. cruzi indicating that THBS1 also plays an important role in the process of cellular infection by T. cruzi. However, the mechanisms by which THBS1 is up-regulated by the parasite to modulate cellular infection are not completely known. The elucidation of T. cruzi surface molecules that interact with THBS1 to enhance cellular infection will advance our understanding of the molecular pathogenesis of T. cruzi infection. We anticipated that NTSP, which is specific to this isoform of thrombospondin, would be essential in the interaction with the parasite because it is different for all thrombospondin isoforms compared to the conserved C-terminal domain (Carlson et al., 2005).

In T. cruzi, it has been suggested that the parasite surface CRT (TcCRT; Ferreira et al., 2004; Aguilar et al., 2005) could play a role in enabling the pathogen to evade the host immune response by interacting with the Clq component of complement (Aguilar et al., 2005). The mechanism by which the parasite CRT interacts with host proteins to enhance the process of cellular invasion remains unknown. We recently hypothesized that T. cruzi uses its surface TcCRT to exploit matricellular proteins regulated by the parasite to enhance cellular infection.

We recently reported that $T$. cruzi up-regulates the expression of THBS1 to enhance the process of cellular invasion. Very recently our group characterized a novel THBS1 interaction with T. cruzi that enhances cellular infection (Johnson et al., 2012). We showed that labeled THBS1 interacts specifically with the surface of T. cruzi trypomastigotes. In Figure 1 we show the full-length THBS1 and the NTSP domain that interact with TcCRT. Pre-exposure of recombinant NTSP or THBS1 to T. cruzi significantly enhanced cellular infection of wild-type mouse embryo fibroblasts (MEF) compared to the C-terminal domain of THBS1, E3T3C1. In addition, blocking TcCRT with antibodies significantly inhibited the 


\section{- (- \\ NTSP \\ E3T3C1}

(aa 1-240; 29kDa)

$\begin{array}{ll}\text { NTSP }=\text { TCCRT Interacting } & \checkmark \text { Type } 1 \text { Repeats } \square \\ \text { Domain } & \square \text { Type 2 Repeats } \quad \bigcirc \quad \begin{array}{l}\text { Type } 3 \text { Repeats } \\ \text { Procollagen Domain }\end{array}\end{array}$

FIGURE 1 |THBS1 structure and NTSP domain that interacts with TcCRT to enhance $T$. cruzi cellular infection.

enhancement of cellular infection mediated by the TcCRT-THBS1 interaction. Taken together, these findings indicate that THBS1 interacts with TcCRT on the surface of T. cruzi through the NTSP domain and that this interaction enhances cellular infection. Thus, surface TcCRT is another virulence factor that enhances the pathogenesis of T. cruzi infection through THBS1, which is up-regulated by the parasite.

The observation that THBS1 interacts with the parasite membrane TcCRT is also in agreement with the recent discovery that CRT, which was previously thought to be exclusively intracellular, is also expressed on the surface of the parasite (Arosa et al., 1999; Xiao et al., 1999; Yan et al., 2010). The identification of CRT homologs in other parasites such as Onchocerca, Schistosoma, and Leishmania (Michalak et al., 1992; Nash et al., 1994; Joshi et al., 1996) suggests that the protein functions as an intracellular chaperone but its role in the process of infection of those parasites remains unknown.

Our group showed that surface TcCRT is a virulence factor that interacts with host THBS1 to enhance cellular infection by T. cruzi (Johnson et al., 2012). In order to explore the significance of host THBS1 and TcCRT in cellular infection by T. cruzi, our group used THBS1 KO MEF and WT MEF in infection assays. We observed that host THBS1 and parasite surface TcCRT are important for MEF cellular infection by T. cruzi. The significance of surface TcCRT in enhancing cellular infection by T. cruzi was supported by the fact that specific antibodies to TcCRT significantly reduced cellular infection. The identification of TcCRT as a virulence factor expressed on the surface of the parasite can be exploited to provide new insights into the molecular pathogenesis of T. cruzi infection. TcCRT expression on the parasite surface may modulate the vertebrate complement system as an immune escape mechanism (Ferreira et al., 2005). Taken together, these findings indicate that THBS1 interacts with TcCRT on the surface of T. cruzi through the NTSP domain and that this interaction enhances cellular infection by T. cruzi.

\section{T. cruzi MODULATES THE THBS1 SUB-NETWORK}

The matricellular THBS1 glycoprotein inhibits angiogenesis and modulates endothelial cell adhesion, motility, and growth. THBS1 interacts with several cell adhesion receptors, including
CD36, integrins, and integrin-associated proteins and inhibits matrix metalloproteinase enzymes thereby remodeling the cellular microenvironment (Sid et al., 2004). We have shown that T. cruzi up-regulates the expression of THBS1 to facilitate the invasion process (Simmons et al., 2006). Knockdown of THBS1 by RNAi significantly inhibited cellular invasion by $T$. cruzi. The increased level of THBS1 expression significantly modulates the interactome cross-talk between the cells, as we found the largest protein-protein interaction (PPI) sub-network occurred in the THBS1 seed network, with 51 nodes interconnected with 151 edges (Nde et al., 2010). This network topology will potentially favor parasite multiplication and survival. During this cross-talk, higher levels of THBS1 activate TGF- $\beta$ (Ribeiro et al., 1999), a major pro-fibrotic cytokine causing modification of the ECM milieu. THBS1 then interacts with several host proteins including the COL7A1 gene product, which then interacts with other proteins from the laminin family of genes to make the ECM conducive to parasite mobility and cellular invasion. The modulation of THBS1 gene networking profile is highly critical for trypanosome mobility and cellular invasion. Other parasites (including the human pathogens Plasmodium, Toxoplasma, and Cryptosporidium), which do not regulate human THBS1, use a transmembrane thrombospondin-related anonymous protein (TRAP) for gliding motility and invasion of vertebrate host cells (Kappe et al., 1999; Sibley, 2004; Morahan et al., 2009). TRAP proteins produced by the parasite can also activate TGF- $\beta$, an anti-inflammatory cytokine that counteracts the effects of inflammatory cytokines like IL-12, INF- $\gamma$, and TNF- $\alpha$ thereby facilitating parasite survival. TSP1, also binds to matrix metalloproteinase-2(MMP-2) and appears to be involved in the infection process.

Modulation of THBS1 expression in HCASM cells by T. cruzi is essential because THBS1 blocks the cytoprotective activity of nitric oxide (NO) by antagonizing the NO/cGMP signaling pathway thereby negatively regulating vascular tone, vascular smooth muscle cells adhesion, chemotaxis, and proliferation (Isenberg et al., 2008). Increased THBS1 transcripts in parasitized HCASM cells may suggest that THBS1 also contributes in part to the pathology caused by T. cruzi. THBS1 is required for the infection process of T. cruzi as evidenced by RNAi of that specific isoform. The upregulation of host THBS1 expression by $T$. cruzi to facilitate the 
infection of human cells represents an additional mechanism that contributes to the pathogenesis of T. cruzi infection.

The first elucidated THBS1 sub-network interactome mobilized in cells by $T$. cruzi involves the following genes: TSC2, TNFRSF1A, COL1A1, COL2A1, COL3A1, COL5A1, COL7A1, SDC1, SDC2, SDC3, SCD4, SPARC, LTBP1, HRG, ITGB1, ITGB3, ITGA3, ITGA4, ITGAV, FGA, SPP1, F2, CD47, MMP2, MMP9, CALR, TFPI, ZNF8, LRP1, LRP5, TP53, CTSG, PDGFB, ELA2, CORO1A, LAMB3, CFH, PDEA, JAG1, DCN, TGFB1, CD36, FN1, PLG, TNFAIP6, SCARB2, DHFR, and KNG1 (Nde et al., 2010). The roles of the members of the THBS1 sub-network in the infection process are also being investigated fully by our group as indicated above in this section. The new advances in the area will facilitate the identification of the molecular signature induced by T. cruzi in cells via the TcCRT-THBS1 interphase as well as the development of small molecule inhibitors to interrupt the critical initial steps of T. cruzi infection.

\section{T. cruzi USES LAMININ AND GALECTIN-3 TO PROMOTE CELLULAR INFECTION}

The ECM, human lectins, and parasite mucins have been shown to play an important role in the early process of $T$. cruzi infection. Accordingly, human galectin-3 binds to a trypomastigote surface mucin (Moody et al., 2000; Turner et al., 2002) and to HCASM cells in a lectin-like manner (Kleshchenko et al., 2004) to significantly increase the adhesion of trypomastigotes to these cells. Silencing galectin-3 expression in cells by antisense approach significantly reduces trypomastigote cell adhesion. Galectin-3 molecules interact with a $T$. cruzi $45 \mathrm{kDA}$ mucin surface protein on one hand and with laminin on the other, via their carbohydrate recognition domains and are joined together through the R-domains (Moody et al., 2000; Kleshchenko et al., 2004). In this way, galectin-3 binds to laminin and trypomastigotes to recruit them to the ECM thus facilitating initial infection. Thus, galectin-3 provides a bridge between parasites and laminin in host cell thereby enhancing infection. Prior to cellular infection the infective trypomastigotes bombard host cells with PLC-cleaved gp83 to activate the ERK1/2 pathway to up regulate laminin primed cells to enhanced infection via the laminin-45 mucin-galectin-3 pathway (Moody et al., 2000; Kleshchenko et al., 2004). These studies pointed out a novel T. cruzi-host cell interaction mediated by gp83-laminin45 mucin- galectin-3 that recruits significant number of parasites at the ECM to facilitate cellular infection. Other T. cruzi surface antigens bind to laminin (Giordano et al., 1999) and fibronectin (Ouaissi et al., 1986) and have been postulated to participate in the infection process, however these interactions have not been studied using systems biology approaches. Overall, we can conclude that the parasite modulates some ECM components and interacts with them to facilitate infection by exploiting these molecules and human lectins to recruit parasites in the early process of infection.

Galectin-3 binds to the surface of HCASM cells in a granular manner, distributed around the cellular membrane, polarized, and more pronounced at the cellular ends (Kleshchenko et al., 2004). The receptors for human galectin-3 are distributed in patches on the cell surface and are more abundant at the cellular terminal regions. The binding of galectin- 3 to trypomastigotes is also seen as granular, restricted to some areas of the trypanosome membrane, and polarized (Kleshchenko et al., 2004).

Galectin-3 is also implicated in the association of T. cruzi trypomastigotes with laminin (Moody et al., 2000). Binding of trypomastigotes to laminin is enhanced by galectin-3 and this enhanced binding of trypanosomes is inhibited by lactose. Coimmunoprecipitations indicate that galectin-3 binds to the 45 $\mathrm{kDa}$ trypomastigote surface protein and this binding is also inhibited by lactose (Moody et al., 2000). The monoclonal antibody B5 that recognizes the trypomastigote $45 \mathrm{kDa}$ surface mucin blocks trypomastigote attachment to heart myoblasts (Moody et al., 2000; Turner et al., 2002). A working model proposes that galectin-3, released by human cells, forms bridges between $T$. cruzi and laminin. Since nearly all the tissues which T. cruzi infects are surrounded by basement membranes of which laminin is a major constituent, its ability to effectively interact with laminin is critically important for passage through the membrane barrier. These studies suggest that this is a trypanosome trapping mechanism, which enables the organisms to accumulate in the basement membrane prior to invasion of heart myoblasts, making galectin-3 a candidate molecule, which enhances the pathogenesis of $T$. cruzi via laminin and a $T$. cruzi surface mucin.

Galectins have long been suspected of modulating cell to ECM interactions in a novel fashion (Liu and Rabinovich, 2005). Data suggest that one mechanism involves the ligation of mammalian cells to ECM proteins, which also interact with galectin-3 such as laminin and elastin (Liu and Rabinovich, 2005). The other mechanism involves the interaction of galectins with the polylactosamine residues of integrins, resulting in the modulation of cellular adhesion to ECM proteins (Rabinovich et al., 2002). Whereas most of these studies were done in mammalian systems, it has been suggested that galectins expressed by Entamoeba may be critical in their interactions with host cells (Petri et al., 1990). It is likely that in parasitic organisms, the binding of the organisms to the ECM proteins or cell surface glycoconjugates may be the primary adhesion mechanisms mediated by galectins.

Galectin-3 is also expressed in B cells from T. cruzi-infected mice (Acosta-Rodríguez et al., 2004) and is up-regulated by $T$. cruzi infection of mice (Vray et al., 2004). The fact that galectin3 is secreted by macrophages and by other cells, including HCASM cells, suggests that released galectin- 3 modulates infection via laminin. The concentrations of galectin-3 that increase trypanosome adhesion to HCASM cells in vitro are similar to the concentrations of galectin-3 present in fluids in vivo (Sano et al., 2000). Furthermore, the concentrations of galectin-3 in fluids in vivo increase approximately 300-fold during microbial infection (Sato and Hughes, 1994). These observations suggest that the parasite may have adapted to the host and that it takes advantage of a host inflammatory molecule, galectin-3, to bind to host cells via laminin and a T. cruzi mucin to enhance infection.

Previous studies have shown that parasite molecules bind to immobilized laminin (Giordano etal., 1999) and that human galectin-3 enhances this interaction (Moody et al., 2000), indicating that the trypanosome interacts with laminin via galectin-3. 


\section{T. cruzi SURFACE GP83 REGULATES THE ECM INTERACTOME}

The genetic architecture of the early T. cruzi infection process of human cells is very limited. To understand this aspect of the infection, we conducted gene transcription microarray analysis followed by gene network construction of the host cell response in primary HCASM cells infected with T. cruzi or exposed to T. cruzi gp83. Using THBS1, LAMC1, LGALS3, and ERK1/2 as the seed nodes for biological network construction, we built an interactome network of the early T. cruzi infection process, which centered on the ECM. ERK1/2 was used as one seed mode, since T. cruzi gp 83 activates ERK1/2 in cells to up-regulate the LAMC1 expression and infection. After seeding the initial four nodes, the network was expanded to one degree of direct biological interaction, resulting in base interaction networks with the seed node as a center point in each. In order to populate and build our interaction network we used a similar approach to determine the modulation of the LAMC1 sub-network as indicated above. Figure 2 shows the ECM interactome that is modulated by T. cruzi gp83 ligand. The first step in the regulation of the ECM interactome by $T$. cruzi during the initial infection process involves triggering gp83 receptors in the cell via activation of ERK1/2 by PLC-cleaved gp83 which results in the up-regulation of LAMC1 and activation of the LAMC1 sub-network which directly cross-talks with the LGALS3 sub-network to recruit parasites and enhance cellular infection on one side. On the other side, triggering gp83 receptors in the cell via ERK1/2 cross-talk with the THBS1 sub-network, also enhances cellular infection and cross-talk at a distance with LGALS3, which also recruit parasites via Tc45 mucin, and LAMC1 (Figure 2). Tc85 interacts with laminin and may mobilize the ECM interactome. It is also possible that T. cruzi MMP9 like

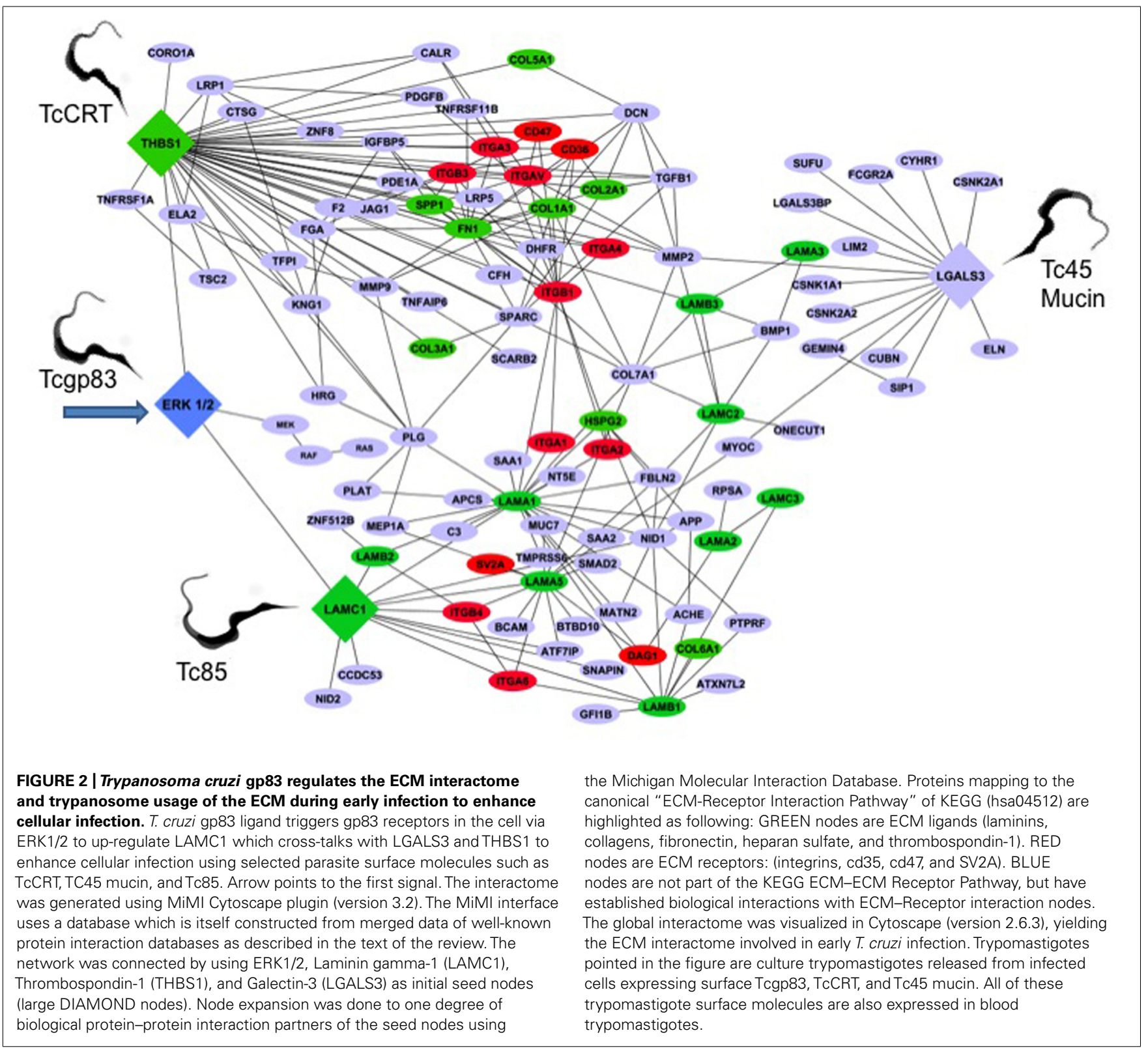


activity that degrades the ECM may regulate the ECM interactome (Nogueira de Melo et al., 2010). This ECM-focused interactome contains 108 nodes representing protein-coding genes connected by 222 edges representing biological interactions between nodes (Figure 2).

Increased THBS1 expression significantly modulates the interactome cross-talk between cells. This change in network topology potentially favors parasite invasion and infection of host cells. As such, THBS1 interacts with several proteins ranging from adhesion receptors (CD36 and CD47) to structural proteins (COL7A1) and zymogens. CD47 is a receptor for the C-terminal domain of THBS1 and this interaction may be important in membrane transport and signal transduction. In addition, CD47 is involved in intracellular calcium increase, which occurs when the cell adheres to the ECM. An increase in cytosolic $\mathrm{Ca}^{++}$in $T$. cruzi trypomastigotes was detected at the single cell level after association of the parasites with myoblasts. $\mathrm{Ca}^{++}$mobilization in host cells was also detected upon contact with trypomastigotes. Confirmatively, pretreatment of the parasites with the $\mathrm{Ca}^{++}$chelators decreased trypomastigote association to myoblasts indicating that calcium mobilization is required for cell invasion (Moreno et al., 1994).

The structural protein COL7A1 is typically found in the basement membrane and also associates with THBS1. Once COL7A1 interacts with THBS1, it makes the ECM conducive to parasite motility and cellular invasion with the help of proteins from the laminin family of genes. Plasminogen (PLG) links LAMC1 and THBS1. PLG (a zymogen) is cleaved into plasmin (a serine protease), and angiostatin (an angiogenesis inhibitor). Plasmin is known to cleave fibronectin, THBS1, and LAMC1, thus taking part in ECM modifications.

Trypanosoma cruzi must navigate through the basal lamina, which contains LAMC1. The T. cruzi gp83 ligand modifies LAMC1 expression in the ECM and contributes to the pathogenesis of T. cruzi infection in human heart cells. The fact that the LAMC1 network is connected to THBS1 through PLG and COL7A1 and matrix metalloproteinase 2 (MMP2) suggests that this network could facilitate parasite mobilization. MMP2 is a type IV collagenase that participates in the rearrangement of the ECM, which could facilitate parasite mobilization. In the LAMC1 sub-network there is an indirect connection (through LAMA1) to dystroglycan (DAG1). DAG1 is a dystrophin-associated glycol-protein responsible for transmembrane linkage between the ECM and host cell cytoskeleton. The extracellular form of DAG1 can bind to merosin alpha-2-laminin in the ECM (Yamada et al., 1994). If T. cruzi alters the dystroglycan complex, it could consequently manipulate or weaken the host cell cytoskeleton prior to gaining entry into the cell. This could yet be another mechanism by which T. cruzi invades host cells. In reference to the ECM network reviewed, LAMC1 also has a second-degree interaction (one node in between the genes) with LGALS3 through myocilin (MYOC). MYOC is a secreted protein believed to have a role in cytoskeletal function, specifically vesicular transport and ECM conformation (Caballero et al., 2000). Unlike other intracellular pathogens, which avoid contact with host cell lysosomes, T. cruzi requires the low-pH environment of lysosomes to initiate egression from the vacuole and delivery to the host cell cytoplasm where replication takes place
(Burleigh, 2005). Therefore, the control of vesicular trafficking by T. cruzi improves the rate of trypomastigote entry and amastigote replication in the host cell. The fact that LAMC1 is connected to LGALS3 through MYOC in the ECM interactome suggests the importance of LGALS3 in the manipulation of host ECM by T. cruzi.

Increased LGALS3 expression in the ECM promotes the adhesion of T. cruzi to host cells and subsequent infection (Kleshchenko et al., 2004). In addition, LGALS3 has numerous ECM interacting partners (Dumic et al., 2006), including collagen IV, hensin, laminins, fibronectin, vitronectin, tenascin, and elastin. LGALS3 regulates adhesion of these ECM proteins to a variety of host cells. Matrix metalloproteases, which are more active in T. cruzi infected mice, regulate LGAL3 function (Gutierrez et al., 2008). When metalloproteases are activated in the ECM, they can cleave LGALS3 and negatively regulate its function. Consequently, increased activation of MMP1 and MMP9 is associated with ECM destruction and myocarditis in T. cruzi infection.

Multiple types of collagen interact with the three central seed nodes, THBS1, LGALS3, and LAMC1. A glimpse of the importance of collagen in early $T$. cruzi infection was reported (Velge et al., 1988).

SOME POTENTIAL IMPLICATIONS OF THE ECM IN INFECTION Expression profiling microarray studies of T. cruzi infected cells have shown that some ECM genes are regulated (Goldenberg et al., 2009; Mukherjee et al., 2003), however those genes were not confirmed by RT-PCR, nor their function in the process of $T$. cruzi infection was determined. Several reports suggest that some parasite proteases may degrade the ECM. The possible role of trypanosomatid surface proteases in parasite survival and infection has been discussed (Marino et al., 2003; Yao, 2010). It was suggested that the gp85/trans-sialidase interacts with the ECM components (Alves and Colli, 2008) and speculated that the prolyl oligopeptidase Tc 80 may be involved in the infection possibly by acting on ECM components (Grellier et al., 2001). Interestingly, it was found that in a T. cruzi infected mouse embryo hepatocyte cell line, there was a reduction of MMP9 (Nogueira de Melo et al., 2004), as well as some matrix components during the late phase of infection (60th to 90th day; Magalhaes-Santos et al., 2002). Pinho et al. (2002) reported that uncharacterized parasite antigens bind to non-infected cells and that ECM components were recognized by antibodies, however the molecular characterization of antigens and the specificity of these possible interactions were not considered. Furthermore, Santana et al. (1997) showed that a Tc 80 proteinase hydrolyzes collagen type in rat mesentery. It is possible that that ECM derived from T. cruzi infected endothelial cells directs phenotypic expression (Morris et al., 1990).

It has been suggested that there are fibrotic implications during T. cruzi infection involving the ECM. Accordingly, Calvet et al. (2009) suggested that TGF- $\beta$ and TNF- $\alpha$ stimulate fibronectin expression in uninfected cells of T. cruzi-infected cultures, whereas cells harboring the parasites display low or no fibronectin fibrils. It was observed that there are sequential changes of the connective matrix components of the myocardium (fibronectin and laminin) during fibrosis in infected mice (Andrade et al., 1989). The fact that 
T. cruzi-mediated down-regulation of CTGF expression requires de novo host cell protein synthesis and that T. cruzi interferes with the host fibrogenic response suggest that this complex process requires input from multiple host cell signaling pathways (Unnikrishnan and Burleigh, 2004). Although it has been demonstrated that cardiomyocytes are able to synthesize cytokines upon T. cruzi infection, Calvet et al. (2004) suggest that matrix remodeling is dependent on cytokines secreted by inflammatory cells recruited in immune response. These reports suggest that T. cruzi modulates the ECM during the infection process with potential implications in the pathology of the disease.

\section{CONCLUDING REMARIS}

Despite an appreciation of the involvement of the ECM in T. cruzi infection, the difficulty in delineating regulatory networks in the process of cellular infection and disease, has until now prevented a comprehensive assessment of T. cruzi infection at the molecular level. The use of systems biology approaches to elucidate the ECM interactome network regulated by T. cruzi and its gp83 ligand that mediate trypanosome attachment and entry are critically important to understand the molecular pathogenesis of T. cruzi infection and to design novel approaches for intervention and disease management. There is an expectation that systems biology approaches applied to understand T. cruzi infection would bring new innovative strategies for the treatment and control of Chagas

\section{REFERENCES}

Acosta-Rodríguez, E. V., Montes, C. L., Motran, C. C., Zuniga, E. I., Liu, F. T., Rabinovich, G. A., et al. (2004). Galectin-3 mediates IL-4-induced survival and differentiation of B cells: functional cross-talk and implications during Trypanosoma cruzi infection. J. Immunol. 172, 493-502.

Aguilar, L., Ramirez, G., Valck, C., Molina, M. C., Rojas A., Schwaeble, W., etal. (2005). F( $\left(\mathrm{ab}^{\prime}\right) 2$ antibody fragments against Trypanosoma cruzi calreticulin inhibit its interaction with the first component of human complement. Biol. Res. 38, 187-195.

Alves, M. J., and Colli, W. (2008). Role of the gp 85/trans-sialidase superfamily of glycoproteins in the interaction of Trypanosoma cruzi with host structures. Subcell. Biochem. 47, 58-69.

Andrade, S. G., Grimaud, J. A., and Stocker-Guerret, S. (1989). Sequential changes of the connective matrix components of the myocardium (fibronectin and laminin) and evolution of cardiac fibrosis in mice infected with Trypanosoma cruzi. Am. J. Trop. Med. Hyg. 40, 252-260.

Arosa, F. A., de Jesus, O., Porto G., Carmo, A. M., and de Sousa, M. (1999). Calreticulin is expressed on the cell surface of activated human peripheral blood $\mathrm{T}$ lymphocytes in association with major histocompatibility complex class I molecules. J. Biol. Chem. 274, 16917-16922.

Bornstein, P. (2002). Cell-matrix interactions: the view from the outside Methods Cell Biol. 69, 7-11.

Burleigh, B. A. (2005). Host cell signaling and Trypanosoma cruzi invasion: do all roads lead to lysosomes? Sci. STKE. 2005, pe36.

Caballero, M., Rowlette, L. L., and Borras, T. (2000). Altered secretion of a TIGR/MYOC mutant lacking the olfactomedin domain. Biochim. Biophys. Acta 1502, 447-460.

Calvet, C. M., Meuser, M., Almeida D., Meirelles, M. N., and Pereira, M. C. (2004). Trypanosoma cruzicardiomyocyte interaction: role of fibronectin in the recognition process and extracellular matrix expression in vitro and in vivo. Exp. Parasitol. 107, 20-30.

Calvet, C. M., Oliveira, F. O. Jr., Araújo-Jorge, T. C., and Pereira, M. C. (2009). Regulation of extracellular matrix expression and distribution in Trypanosoma cruzi-infected cardiomyocytes. Int. J. Med. Microbiol. 299, 301-312.

Cardenas, T., Pratap, S., Nde, P. N., Johnson, C., Kleshchenko, Y. Y., Furtak, Y., et al. (2010). Regulation of the extracellular matrix interactome by Trypanosoma cruzi. Open Parasitol. J. 4, 72-76.

disease. So far the use of systems biology to understand the role of the ECM in T. cruzi infection has significantly enhanced the understanding of T. cruzi-host cell interaction and pathogenesis of T. cruzi infection. Accordingly, here we elucidate the first ECM interactome triggered by the T. cruzi gp83 ligand during the first step of cellular infection to recruit trypanosomes at the ECM to enhance cellular infection. In the shot gun used by T. cruzi to regulate the ECM interactome to gain cellular entry and evade the host reported here, $T$. cruzi gp 83 triggers gp 83 receptors in the cell via ERK1/2 to up-regulate LAMC1 which cross-talks with both LGALS3 and THBS1 to enhance cellular infection using selected parasite surface molecules such as TcCRT and TC45 mucin. Based on this new information, current efforts are being pursued by our group to elucidate the molecular signature induced by T. cruzi in cells. Since T. cruzi-host cell interaction is of a complex nature, the elucidation of the global interactome and sub-interactome networks will help understand the complex T. cruzi-host interphase that mediate parasite attachment and entry, and develop new means for the treatment of the highly neglected and complex Chagas disease, which has no current effective drugs or vaccines.

\section{ACKNOWLEDGMENTS}

This work was supported primarily by a NIH grant AI080580 and in part by NIH grants AI007281, AI083925, U54 MD007593, GM059994, and MD007586.

Carlson, C. B., Bernstein, D. A., Annis, D. S., Misenheimer, T. M., Hannah, B. L., Mosher, D. F., et al. (2005). Structure of the calcium-rich signature domain of human thrombospondin2. Nat. Struct. Mol. Biol. 12, 910-914.

Chen, H., Herndon, M. E., and Lawler, J. (2000). The cell biology of thrombospondin-1. Matrix Biol. 19, 597-614.

Cline, M. S., Smoot, M., Cerami, E., Kuchinsky, A., Landys, N., Workman, C., et al. (2007). Integration of biological networks and gene expression data using Cytoscape. Nat. Protoc. 2 , 2366-2382.

Dumic, J., Dabelic, S., and Flogel, M. (2006). Galectin-3: an open-ended story. Biochim. Biophys. Acta 1760, 616-635.

Elzie, C. A., and Murphy-Ullrich, J. E. (2004). The N-terminus of thrombospondin: the domain stands apart. Int. J. Biochem. Cell Biol. 36, 10901101.

Ferreira, V., Molina, M. C., Schwaeble, W., Lemus, D., and Ferreira, A. (2005). Does Trypanosoma cruzi calreticulin modulate the complement system and angiogenesis? Trends Parasitol. 21, 169-174.

Ferreira, V., Molina, M. C., Valck, C., Rojas, A., Aguilar, L., Ramirez, G., et al. (2004). Role of calreticulin from parasites in its interaction with vertebrate hosts. Mol. Immunol. 40, 1279-1291.
Gao, A. G., and Frazier, W. A. (1994) Identification of a receptor candidate for the carboxyl-terminal cell binding domain of thrombospondins. J. Biol. Chem. 269, 29650-29657.

Gao, J., Ade, A. S., Tarcea, V. G., Weymouth, T. E., Mirel, B. R., Jagadish, H. V., et al. (2009). Integrating and annotating the interactome using the MiMI plugin for cytoscape. Bioinformatics 25, 137-138.

Giordano, R., Fouts, D. L., Tewari, D., Colli, W., Manning, J. E., and Alves, M. J. (1999). Cloning of a surface membrane glycoprotein specific for the infective form of Trypanosoma cruzi having adhesive properties to laminin. J. Biol. Chem. 274, 34613468.

Gold, L. I., Eggleton, P., Sweetwyne, M. T., Van Duyn, L. B., Greives, M. R., Naylor, S. M., et al. (2010). Calreticulin: non-endoplasmic reticulum functions in physiology and disease. FASEB J. 24, 665-683.

Goldenberg, R. C., Iacobas, D. A., Iacobas, S., Rocha, L. L., da Silva de Azevedo Fortes, F., Vairo, L., et al. (2009). Transcriptomic alterations in Trypanosoma cruzi-infected cardiac myocytes. Microbes Infect. 11, 1140-1149.

Grellier, P., Vendeville, S., Joyeau, R., Bastos, I. M., Drobecq, H., Frappier, F., et al. (2001). Trypanosoma cruzi prolyl oligopeptidase Tc80 is involved in nonphagocytic mammalian cell 
invasion by trypomastigotes. J. Biol. Chem. 276, 47078-47086.

Gutierrez, F. R., Lalu, M. M., Mariano, F. S., Milanezi, C. M., Cena, J., Gerlach, R. F., et al. (2008). Increased activities of cardiac matrix metalloproteinases matrix metalloproteinase (MMP)-2 and MMP-9 are associated with mortality during the acute phase of experimental Trypanosoma cruzi infection. J. Infect. Dis. 197, 1468-1476.

Isenberg, J. S., Maxhimer, J. B., Hyodo, F., Pendrak, M. L., Ridnour, L. A., DeGraff, W. G., et al. (2008). Thrombospondin-1 and CD47 limit cell and tissue survival of radiation injury. Am. J. Pathol. 173, 1100-1112.

Johnson, C. A., Kleshchenko, Y. Y., Ikejiani, A. O., Udoko, A. N., Cardenas, T. C., Pratap, S., et al. (2012). Thrombospondin-1 interacts with Trypanosoma cruzi surface calreticulin to enhance cellular infection. PLOS ONE 7, e40614. doi: 10.1371/journal.pone.0040614

Johnson, R. J., Xiao, G., Shanmugaratnam, J., and Fine, R. E. (2001a). Calreticulin functions as a molecular chaperone for the beta-amyloid precursor protein. Neurobiol. Aging 22, 387-395.

Johnson, S., Michalak, M., Opas, M., and Eggleton, P. (2001b). The ins and outs of calreticulin: from the ER lumen to the extracellular space. Trends Cell Biol. 11, 122-129.

Joshi, M., Pogue, G. P., Duncan, R. C., Lee, N. S., Singh, N. K., Atreya, C. D., et al. (1996). Isolation and characterization of Leishmania donovani calreticulin gene and its conservation of the RNA binding activity. Mol. Biochem. Parasitol. 81, 53-64.

Kappe, S., Bruderer, T., Gantt, S., Fujioka. H., Nussenzweig, V., and Menard, R. J. (1999). Conservation of a gliding motility and cell invasion machinery in Apicomplexan parasites. J. Cell Biol. 147, 937-944.

Kleshchenko, Y. Y., Moody, T. N., Furtak, F. A., Ochieng, J., Lima, M. F., and Villalta, F. (2004). Human galectin-3 promotes Trypanosoma cruzi adhesion to human coronary artery smooth muscle cells. Infect. Immun. 72, 6717-6721.

Lawler, J., and Hynes, R. O. (1989). An integrin receptor on normal and thrombasthenic platelets that binds thrombospondin. Blood 74, 20222027.

Lima, M. F., and Villalta, F. (1988). Host-cell attachment by Trypanosoma cruzi: identification of an adhesion molecule. Biochem. Biophys. Res. Commun. 155, 256-262.
Lima, M. F., and Villalta, F. (1989). Trypanosoma cruzi trypomastigote clones differentially express a cell adhesion molecule. Mol. Biochem. Parasitol. 33, 159-170.

Liu, F. T., and Rabinovich, G. A. (2005). Galectins as modulators of tumor progression. Nat. Rev. Cancer 5, 29-41.

Magalhaes-Santos, I. F., Lima, E. S., and Andrade, S. G. (2002). Fibrogenesis and collagen resorption in the heart and skeletal muscle of Calomys callosus experimentally infected with Trypanosoma cruzi: immunohistochemical identification of extracellular matrix components. Mem. Inst Oswaldo Cruz 97, 703-710.

Marino, A. P., Silva, A. A., Pinho, R. T., and Lannes-Vieira, J. (2003). Trypanosoma cruzi infection: a continuous invader-host cell cross talk with participation of extracellular matrix and adhesion and chemoattractant molecules. Braz. J. Med. Biol. Res. 36, 1121-1133.

Michalak, M., Milner, R. E., Burns, K. and Opas, M. (1992). Calreticulin. Biochem. J. 285, 681-692.

Michalak, M., Corbett, E. F., Mesaeli, N., Nakamura, K., and Opas, M. (1999). Calreticulin: one protein, one gene, many functions. Biochem. J. 2, 281-292.

Milei, J., Sanchez, J., Storino, R., Yu, Z. X., Denduchis, B., and Ferrans, V. J. (1993). Antibodies to laminin and immunohistochemical localization of laminin in chronic chagasic cardiomyopathy: a review. Mol. Cell. Biochem. 129, 161-170.

Moody, T., Ochieng, J., and Villalta, F. (2000). Novel mechanism that Trypanosoma cruzi uses to adhere to the extracellular matrix-mediated by human galectin-3. FEBS Lett. 470, 305-308.

Morahan, B. J., Wang, L., and Coppel, R. L. (2009). No TRAP, no invasion. Trends Parasitol. 25, 77-84.

Moreno, S. N., Silva, J., Vercesi, A. E., and Docampo, R. (1994). Cytosolic-free calcium elevation in Trypanosoma cruzi is required for cell invasion. J. Exp. Med. 180, 15351540.

Morris, S. A., Wittner, M., Weiss, L., Hatcher, V. B., Tanowitz, H. B., Bilezikian, J. P., et al. (1990). Extracellular matrix derived from Trypanosoma cruzi infected endothelial cells directs phenotypic expression. J. Cell. Physiol. 145, 340-346.

Mukherjee, S., Belbin, T. J., Spray, D. C., Iacobas, D. A., Weiss, L. M., Kitsis, R. N., et al. (2003). Microarray analysis of changes in gene expression in a murine model of chronic chagasic cardiomyopathy. Microarray analysis of changes in gene expression in a murine model of chronic chagasic cardiomyopathy. Parasitol. Res. 91, 187-196.

Nakhasi, H. L., Pogue, G. P., Duncan, R. C., Joshi, M., Atreya, C. D., Lee, N. S., et al. (1998). Implications of calreticulin function in parasite biology. Parasitol. Today 14, 157-160.

Nash, P. D., Opas, M., and Michalak, M. (1994). Calreticulin: not just another calcium-binding protein. Mol. Cell. Biochem. 135, 71-78.

Nde, P. N., Simmons, K. J., Kleshchenko, Y. Y., Pratap, S., Lima, M. F. and Villalta, F. (2006). Silencing of the laminin gamma-1 gene blocks Trypanosoma cruzi infection. Infect. Immun. 74, 1643-1648.

Nde, P. N., Johnson, C. A., Pratap, P., Cardenas, T. C., Kleshchenko, Y. Y., Furtak, Y. A., et al. (2010). Gene network analysis during early infection of human coronary artery smooth muscle cells by Trypanosoma cruzi and its gp83 ligand. Chem. Biodivers. 7, 1051-1064.

Nogueira de Melo, A. C., Meirelles, M. N., Porrozi, R., Costa, J. D., Branquinha, M. H., and Vermelho, A. B. (2004). Reduced activity of matrix metalloproteinase-9 in Trypanosoma cruzi-infected mouse embryo hepatocyte cell. Hepatol. Res. 91, 187-196. Nogueira de Melo, A. C., de Souza, E. P., Elias, C. G., dos Santos, A. L., Branquinha, M. H., d'Avila-Levy, C. M., dos Reis, F. C., et al. (2010). Detection of matrix metallopeptidase-9like proteins in Trypanosoma cruzi. Exp. Parasitol. 125, 256-263.

Ouaissi, M. A., Cornette, J., and Capron, A. (1986). Identification and isolation of Trypanosoma cruzi trypomastigote cell surface protein with properties expected of a fibronectin receptor. Mol. Biochem. Parasitol. 19, 201-211.

Petri, W. A., Snodgrass, T. L., Jackson, T. F., Gathiram, V., Simjee, A. E., Chadee, K., et al. (1990). Monoclonal antibodies directed against the galactose-binding lectin of Entamoeba histolytica enhance adherence. J. Immunol. 144, 4803-4809.

Pinho, R. T., Vannier-Santos, M. A., Alves, C. R., Marino, A. P., Castello Branco, L. R., and Lannes-Vieira, J. (2002). Effect of Trypanosoma cruzi released antigens binding to non-infected cells on anti-parasite antibody recognition and expression of extracellular matrix components. Acta Trop. 83, 103-115.

Rabinovich, G. A., Baum, L. G., Tinari, N., Paganelli, R., Natoli, C., Liu, F. T., et al. (2002). Galectins and their ligands: amplifiers, silencers or tuners of the inflammatory response. Trends Immunol. 23, 313-320.

Ribeiro, S. M., Poczatek, M., SchultzCherry, S., Villain, M., and MurphyUllrich, J. E. (1999). The activation sequence of thrombospondin-1 interacts with the latency-associated peptide to regulate activation of latent transforming growth factorbeta. J. Biol. Chem. 274, 1358613593.

Sano, H., Hsu, D. K., Yu, L., Apgar, J. R., Kuwabara, I., Yamanaka, T., et al. (2000). Human galectin-3 is a novel chemoattractant for monocytes and macrophages. J. Immunol. 165, 2156-2164.

Santana, J. M., Grellier, P., Schrével, J., and Teixeira, A. R. (1997). A Trypanosoma cruzi-secreted $80 \mathrm{kDa}$ proteinase with specificity for human collagen types I and IV. Biochem. J. 325, 129-137.

Sasaki, T., Fassler, R., and Hohenester, E. (2004). Laminin: the crux of basement membrane assembly. J. Cell Biol. 164, 959-963.

Sato, S., and Hughes, R. C. (1994). Regulation of secretion and surface expression of Mac-2, a galactosidebinding protein of macrophages. J. Biol. Chem. 269, 4424-4430.

Sibley, L. D. (2004). Intracellular parasite invasion strategies. Science 304, 248-253.

Sid, B., Sartelet, H., Bellon, G., El Btaouri, H., Rath, G., Delorme, N., et al. (2004). Thrombospondin 1: a multifunctional protein implicated in the regulation of tumor growth. Crit. Rev. Oncol. Hematol. 49, 245-258.

Simmons, K. J., Nde, P. N., Kleshchenko, Y. Y., Lima, M. F., and Villalta. F. (2006). Stable RNA interference of host thrombospondin-1 blocks Trypanosoma cruzi infection. FEBS Lett. 580, 2365-2370.

Turner, C. W., Lima, M. F., and Villalta, F. (2002). Trypanosoma cruzi uses a $45 \mathrm{kDa}$ mucin for adhesion to mammalian cells. Biochem. Biophys. Res. Commun. 290, 29-34.

Unnikrishnan, M., and Burleigh, B. A. (2004). Inhibition of host connective tissue growth factor expression: a novel Trypanosoma cruzi-mediated response. FASEB J. 18, 1625-1635.

Velge, P., Ouaissi, M. A., Cornette, J., Afchain, D., and Capron, A. (1988). Identification and isolation of Trypanosoma cruzi trypomastigote collagen-binding proteins: possible role in cell-parasite interaction. Parasitology 97, 255-268.

Villalta, F., Lima, M. F., Zhou, L., and Ruiz-Ruano, A. (1992). Attachment of Trypanosoma cruzi to host cells: 
a monoclonal antibody recognizes a trypomastigote stage-specific epitope on the gp83 required for parasite attachment. Biochem. Biophys. Res. Commun. 182, 6-13.

Villalta, F., Zhang, Y., Bibb, E. K., Burns, J. M. Jr., and Lima, M. F. (1998). Signal transduction in human macrophages by gp 83 ligand of Trypanosoma cruzi: trypomastigote gp83 ligand up regulates trypanosome entry through the MAP kinase pathway. Biochem. Biophys. Res. Commun. 249, 247-252.

Villalta, F., Zhang, Y., Bibb, K. E., Pratap, S., Burns, J. M. Jr., and Lima, M. F. (1999). Signal transduction in human macrophages by gp 83 ligand of Trypanosoma cruzi: trypomastigote gp83 ligand up-regulates trypanosome entry through protein kinase C activation. Mol. Cell Biol. Res. Commun. 2, 64-70.
Villalta, F., Smith, C. M., Ruiz-Ruano, A., and Lima, M. F. (2001). A ligand that Trypanosoma cruzi uses to bind to mammalian cells to initiate infection. FEBS Lett. 505, 383-388.

Villalta, F., Madison, M. N., Nde, P. N., Kleshchenko Y. Y., and Lima, M. F. (2008). Molecular analysis of early host cell infection by Trypanosoma cruzi. Front. Biosci. 13, 3714-3734.

Vray, B., Camby, I., Vercruysse, V., Mijatovic, T., Bovin, N. V., RicciardiCastagnoli, P., et al. (2004). Upregulation of galectin-3 and its ligands by Trypanosoma cruzi infection with modulation of adhesion and migration of murine dendritic cells. Glycobiology 7, 647-657.

Xiao, G., Chung, T. F., Fine, R. E., and Johnson, R. J. (1999). Calreticulin is transported to the surface of NG10815 cells where it forms surface patches and is partially degraded in an acidic compartment. J. Neurosci. Res. 58, 652-662.

Yamada, H., Shimizu, T., Tanaka, T., Campbell, K. P., and Matsumura, K. (1994). Dystroglycan is a binding protein of laminin and merosin in peripheral nerve. FEBS Lett. 352, 49-53.

Yan, Q., Murphy-Ullrich, J. E., and Song, Y. (2010). Structural insight into the role of thrombospondin-1 binding to calreticulin in calreticulininduced focal adhesion disassembly. Biochemistry 49, 3685-3694.

Yao, C. (2010). Major surface protease of trypanosomatids: one size fits all? Infect. Immun. 78, 22-31.

Conflict of Interest Statement: The authors declare that the research was conducted in the absence of any commercial or financial relationships that could be construed as a potential conflict of interest.
Received: 11 September 2012; paper pending published: 26 September 2012; accepted: 22 October 2012; published online: 06 November 2012.

Citation: Nde PN, Lima MF, Johnson $C A$, Pratap $S$ and Villalta $F$ (2012) Regulation and use of the extracellular matrix by Trypanosoma cruzi during early infection. Front. Immun. 3:337. doi: 10.3389/fimmu.2012.00337

This article was submitted to Frontiers in Microbial Immunology, a specialty of Frontiers in Immunology.

Copyright (c) 2012 Nde, Lima, Johnson, Pratap and Villalta. This is an openaccess article distributed under the terms of the Creative Commons Attribution License, which permits use, distribution and reproduction in other forums, provided the original authors and source are credited and subject to any copyright notices concerning any third-party graphics etc. 